\title{
LA DIVERSIDAD EN SISTEMAS DE PARTIDOS
}

\section{Francisco Derqui Civera}

El presente trabajo arranca de la analogía de situaciones que se da entre las especies vegetales, que compiten por colonizar un espacio físico, y los partidos políticos, que compiten por ocupar un espacio electoral. Cabría, pues, enmarcarlo como una humilde aportación a una «Teoría General de los Sistemas», de la que sus partidarios esperan llegue a unificar las llamadas Ciencias Humanas y las de la Naturaleza. La analogía se establece por cuanto en ambos casos se trata de lo que llamaremos en general «Entidades» (Partidos y Especies), que se componen de un gran número de individuos o unidades iguales, o funcionalmente equivalentes. La competencia entre tales Entidades establece una serie de regularidades en las proporciones con las que se distribuyen el espacio disponible, y en la variación de dichas proporciones en el tiempo. En algunos casos particulares y muy simplificados, como es el que describiremos, tales regularidades pueden ser comunes a muy diferentes niveles, como son el social y el ecológico, y pueden permitir, como se verá, la elaboración de sencillos modelos dinámicos que den cuenta de ellas.

Se denomina Sucesión Ecológica al fenómeno por el cual un territorio virgen es colonizado a lo largo del tiempo por las distintas especies biológicas que están en condiciones de prosperar en dicho territorio. Cuando ello ocurre por primera vez se la llama Sucesión Primaria; sería el caso de un terreno que materialmente aparece a disposición de los seres vivos. Se la llama Sucesión 
Secundaria cuando se trata de un espacio que ya había estado colonizado y que, en un momento dado, queda en situación de volver a serlo. Sería éste el caso de un terreno (Biotopo) del que han quedado eliminadas las especies que lo colonizaban (Biocenosis) a causa de una catástrofe cualquiera, como un incendio; o de la acción humana, como sería una tierra de labor que queda abandonada. Así definidas, el caso más estudiado es el de la Sucesión Secundaria, debido a su mayor accesibilidad. De hecho, una Sucesión Primaria propiamente dicha es cuestión complejísima y más asimilable a la de la evolución en general.

La diferencia fundamental a considerar entre ambos tipos de sucesiones estriba en que en la S. Secundaria, en el momento de iniciarse, ya están potencialmente presentes en el terreno las especies que, al realizar esa potencialidad, entrarán en competencia. Esta distinción refuerza la comparación que se pretende hacer con los partidos políticos, pues, en efecto, podríamos denominar «Sucesión Política» a la ocupación de un espacio electoral por los distintos partidos a lo largo del tiempo, calificándola de Primaria si ello ocurre por primera vez o Secundaria si ocurre a partir de algún acontecimiento que en un momento dado lo pone disponible. Análogamente, el caso de una S. Política Primaria sería objeto en todo caso de la Historia Política, interesándonos en el presente trabajo exclusivamente por la S. Política Secundaria, en la que, en el momento de iniciarse, ya están presentes de forma latente los distintos partidos que entrarán en competencia. Por otro lado, no hay que olvidar que el «terreno» considerado está rodeado de un entorno, con el que interacciona formando un sistema más amplio. La presencia latente de las distintas Entidades en el espacio a "re-colonizar» estará condicionada por ese entorno, lo mismo para el caso de los partidos políticos que para el de las especies vegetales.

Uno de los parámetros más reveladores en el estudio de la Sucesión Ecológica es el concepto de Diversidad $(\bar{H})$. Su significado es perfectamente explícito en la misma palabra. Se trata de un parámetro que «mide» la complejidad de una situación compuesta de diversas clases de unidades, por ejemplo de individuos que pueden pertenecer a diversas especies biológicas, en base a las proporciones relativas de cada una de las clases. Tal parámetro ha sido definido de muchas formas, pero la que parece más afortunada es la que usa la fórmula de Shannon para medir el contenido informativo de un mensaje compuesto de un número limitado de signos:

$$
\bar{H}=-\sum_{i=1}^{N} p_{i} \operatorname{Lg}_{2} p_{i}
$$

siendo $p_{i}$ la proporción relativa con la que está presente el signo $i$, y $N$ el número total de signos. 
Considerando a cada una de las especies que conviven en un Ecosistema como el signo de un mensaje, la aplicación de la fórmula a sus proporciones relativas nos dará la medida en bits de la incertidumbre asociada a la identidad de un individuo tomado al azar en aquel espacio. Es decir, una medida de la diversidad de especies en aquel Ecosistema.

Establecido el concepto, los ecólogos se encontraron con que la Diversidad específica de cada uno de los Sistemas Naturales es notablemente constante, y que varía en un estrecho margen, no siendo casi nunca superior a los 5 bits/ individuo. Por otro lado, es tanto mayor cuanto mejores son las condiciones para el prosperar de los seres vivos, la más decisiva de las cuales es, sin duda, la disponibilidad de Energía. Así, se encuentra que la Diversidad de las selvas tropicales es mayor que la de los bosques templados, y la de éstos mayor que la de las tundras árticas. Ello es intuitivamente entendible si se piensa que la vida consiste en estructuras materiales que se mantienen dinámicamente contra la degradación (impuesta por el segundo principio de la Termodinámica) usando la energía disponible de su entorno.

Por otro lado, se admite, aunque no sin polémica, que una de las características de los Sistemas Vivos considerados globalmente es su tendencia evolutiva a aumentar su complejidad, lo que implica aumentar su Diversidad. Conceptuándola como se ha hecho en el párrafo anterior, podría entenderse que la vida tiende a aumentar su propia eficacia, es decir, a mantener «más vida» con las mismas disponibilidades. Se trata, pues, de una característica muy -significativa. Y parece cumplirse no sólo para los Sistemas Naturales globales, sino también para cada uno de los Subsistemas que lo componen, siempre y cuando no estén condicionados (interactuando), como tales, con otros sectores del Sistema global. Por ejemplo, los Mamíferos o los Cormófitos de un Ecosistema tenderán a diversificarse más y más con la evolución. Pero decíamos que muy significativa, porque esta tendencia parece advertirse también en los Sistemas Sociales y/o Políticos, lo que ha alimentado puntos de vista que los consideran como Seres Vivos; es el caso de la Sociología de H. Spencer o de la Filosofía de la Historia de A. Toynbee.

Aplicada a lo largo de una Sucesión Secundaria, sería de esperar que la Diversidad del Sistema incipiente fuese sencillamente aumentando hasta alcanzar el valor máximo que aquellas condiciones pueden sostener, que serían los del entorno. $Y$, en efecto, así sucede de forma más o menos asintótica, excepto en los compases iniciales de la Sucesión, en los que con frecuencia se encuentran diversidades anormalmente altas y, además, con tendencia a disminuir. Este fenómeno se corresponde con una imagen muy familiar en cualquier ciudad: la de los solares y desmontes abandonados, que frecuentemente presentan una vegetación exuberante, más de lo que sería la vegetación «natural» en aquel paisaje. La explicación de estas Diversidades «anormalmente altas» iniciales habría que verla en la ausencia de competencia que hay en el 
instante inicial de «abandono» del terreno, que propicia la aparición de cuanta especie esté en forma de simiente, es decir, en forma latente, y sobre todo de las llamadas "especies oportunistas", cuya estrategia se basa precisamente en una alta tasa de reproducción, lo que hace que sus semillas y propagadores se encuentren en abundancia en cualquier parte. El que estas especies, además, tengan un ciclo vital muy rápido favorece su rápida renovación, y la aparición de nuevas especies.

Podemos resumir todo esto en una gráfica de la evolución de la Diversidad a lo largo del tiempo, lo que daría lugar a un pequeño modelo del inicio de una Sucesión Secundaria, que aplicaremos posteriormente al caso político (gráfico 1). En dicha gráfica distinguiremos tres fases:

Fase I. Que podríamos denominar «Explosiva» y que, según las características dinámicas del sistema, puede ser rapidísima, e incluso inmediata. Como ya hemos dicho, en ella ocurre que en el momento inicial del abandono, al no haber competencia ni haberse puesto de manifiesto mecanismo alguno de restricción, pues el sistema aún no está constituido, brotan cuantas especies estén en condiciones de hacerlo, dando lugar a una «explosión» de la Diversidad a valores «anormalmente altos».

En el caso político, esta situación inicial podría equivaler a la institucionalización en un momento dado de un sistema de representación parlamentaria. Cuando esto sucede, y en España tenemos fresca memoria de tal suceso, saltan a la palestra cuanta ideología hubiera previamente en el cuerpo social. El

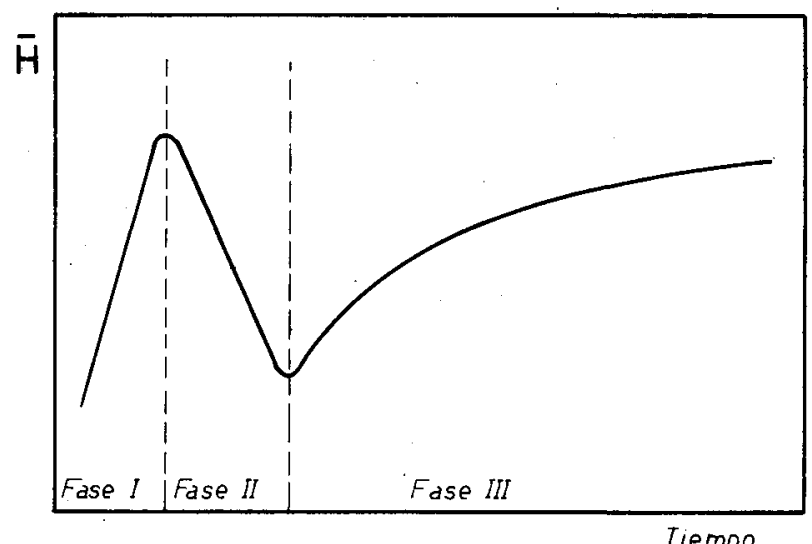

Grático 1.-Esquema general de la variación de la Diversidad $(\bar{H})$ en el inicio de una Sucesión Secundaria. 
resultado es que el electorado ( $\sin$ saber aún a qué atenerse) se reparte en un espectro amplio y variado de partidos cuya competitividad está aún por ver.

Fase II. Que se inicia en cuanto el Sistema adquiere características de tal, con interacciones múltiples entre las entidades en competencia. Podríamos llamarla «Fase de Ajuste», porque durante la misma la Diversidad decrece hacia valores más acordes con el inicio de una Sucesión. Sería ésta la fase en la que las especies, o entidades, que inicialmente brotaron de forma desordenada y azarosa, se van haciendo sitio unas a costa de las otras, y las más competitivas van desplazando a las que van quedando «apeadas» de la Sucesión. La Diversidad, pues, va disminuyendo hasta un valor mínimo, a partir de cuyo momento ya no desaparecen más especies, o su ritmo de desaparición se iguala con el de llegada de otras nuevas.

El equivalente político de esta fase podría ser lo que se conoce como «bipolarización». Cuando la primera fase es inmediata, ésta es la situación con la que se inicia la Sucesión, como veremos que ocurre con los Sistemas políticos de los que nos ocuparemos, en los que de entrada aparecen altas diversidades, y con tendencia inmediata a decrecer.

Fase III. En la que, alcanzada una diversidad mínima, ésta vuelve a crecer asintótica e indefinidamente. Podríamos llamarla «Fase de Madurez», puesto que en ella se pone de manifiesto positivamente la dinámica propia de los Sistemas Vivos tal y como la hemos descrito, es decir, implicando un aumento progresivo de la complejidad del Sistema. Ya dijimos que se ha polemizado mucho sobre tal fenómeno, pero la explicación dinámica más general aceptada por los ecólogos es que consiste en el desplazamiento de las especies «oportunistas» por las «especialistas». Es decir, que las especies capacitadas para sobrevivir en un amplio espectro de condiciones vitales van siendo desplazadas por especies más eficaces en la explotación de unas condiciones dadas, aunque menos flexibles. Esta especialización cada vez mayor va dejando huecos de recursos sin explotar, lo que propicia la llegada de nuevos especialistas. Este mecanismo, que podría prolongarse indefinidamente, se encuentra empíricamente limitado, como ya dijimos. Parece como si, en un momento dado de la evolución, los sistemas no puedan soportar más que un cierto grado de diversidad, hacia el que tienden asintomáticamente y sobre el que fluctúan bajo la concurrencia de otros factores.

Con este marco conceptual trazado, podemos ya abordar los datos concretos de este trabajo.

El aspecto estrictamente biológico de este esquema de acontecimientos descrito para la Sucesión es fenómeno muy conocido para los ecólogos, habiendo sido formulado por vez primera en la Teoría sobre Insularidad de MacArthur y Wilson. Un ejemplo muy explícito es el trabajo de F. A. Baz- 
$z a{ }^{1}$. En él se expone una gráfica de la diversidad a lo largo de la sucesión cuyo ajuste al modelo expuesto no necesita de mayor comentario (gráfico 2 ).

Para la aplicación del modelo a sucesiones políticas se tomaron series de resultados electorales de cuatro países, ateniéndose simplemente a la accesibilidad y manejabilidad de los datos de la obra de Dieter Nohlen ${ }^{2}$.

Con excepción del caso español, cuyos datos proceden de los publicados por la prensa.

Para su manejo hubo que recurrir a algunas simplificaciones. En primer lugar, se trata de series a partir de la Segunda Guerra Mundial. Para los países que participaron en la misma se considera el acontecimiento como equivalente al abandono de un Ecosistema a su propia dinámica, porque durante la guerra hay que suponer que el Sistema Político está condicionado en un contexto más amplio. Finalizada ésta es cuando puede decirse que el Sistema empieza a evolucionar con su propia dinámica en un contexto que el tiempo transcurrido ha hecho «nuevo».

La otra simplificación, también impuesta por la forma de los datos, consiste en obviar la presencia de los partidos minoritarios, frecuentemente agrupados bajo la denominación «otros». La fórmula matemática empleada permite hacerlo sin perder demasiada significación, puesto que se promedia

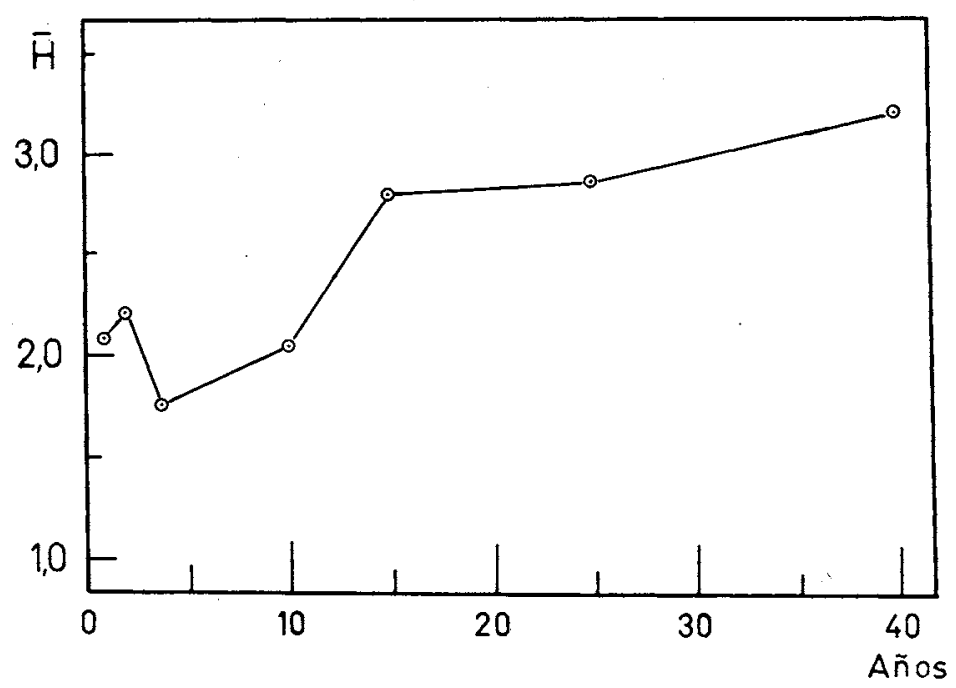

Gráfico 2.-Relación entre Diversidad de especies vegetales $(\bar{H})$, y duración de la sucesión en años de abandono, en campos abandonados en el sur de lllinois. De F. A. Bazzaz, «Plant Species Diversity in Old-Field Successional Ecosystems in Southern Illinois», Ecology, 1975, vol. 56, núm. 2, p. 486.

' F. A. Bazzaz, «Plant Species Diversity in Old-Field Successional Ecosystems in Southern Illinois», Ecology, 1975, vol. 56, núm. 2, p. 486.

2 Dieter No h LEN, Sistemas electorales del mundo, Centro de Estudios Constitucionales. 
$\left(\Sigma p_{i} \ldots\right)$ el grado de incertidumbre $\left(\ldots \operatorname{Lg}_{2} p_{i}\right)$ aportado por cada entidad. Ello hace que el valor global quede decidido por los tres o cuatro partidos que normalmente se reparten el electorado. Haciendo los cálculos en base a las proporciones entre ellos, se obtiene una idea razonablemente fidedigna de la evolución de la diversidad global, aunque su valor absoluto no sea más que aproximado.

Con estas puntualizaciones podemos ver ya, en primer lugar, el caso de Gran Bretaña, en sus elecciones a la Cámara Baja (gráfico 3). El resultado, como se ve, se ajusta al modelo incluso más de lo que era razonablemente de esperar. Destacan en él los bajos valores globales y la rápida evolución del electorado, lo que hace pensar en una situación previa ya bastante "madura», en el sentido de que el Sistema Político, aunque condicionado por la guerra, no fue barrido por ella.

En contraposición al anterior, el caso alemán, en sus elecciones al Bundestag (gráfico 4), presenta unos valores iniciales más altos y una evolución posterior más lenta. Una primera interpretación podría considerar que en este caso el Sistema Político sí fue literalmente eliminado, lo que provoca una situación inicial más azarosa y un mayor tiempo de «ajuste». Este período se alarga hasta el año 76 (casi treinta años de polarización creciente), en el que parece iniciarse la que llamamos «Fase de Madurez».

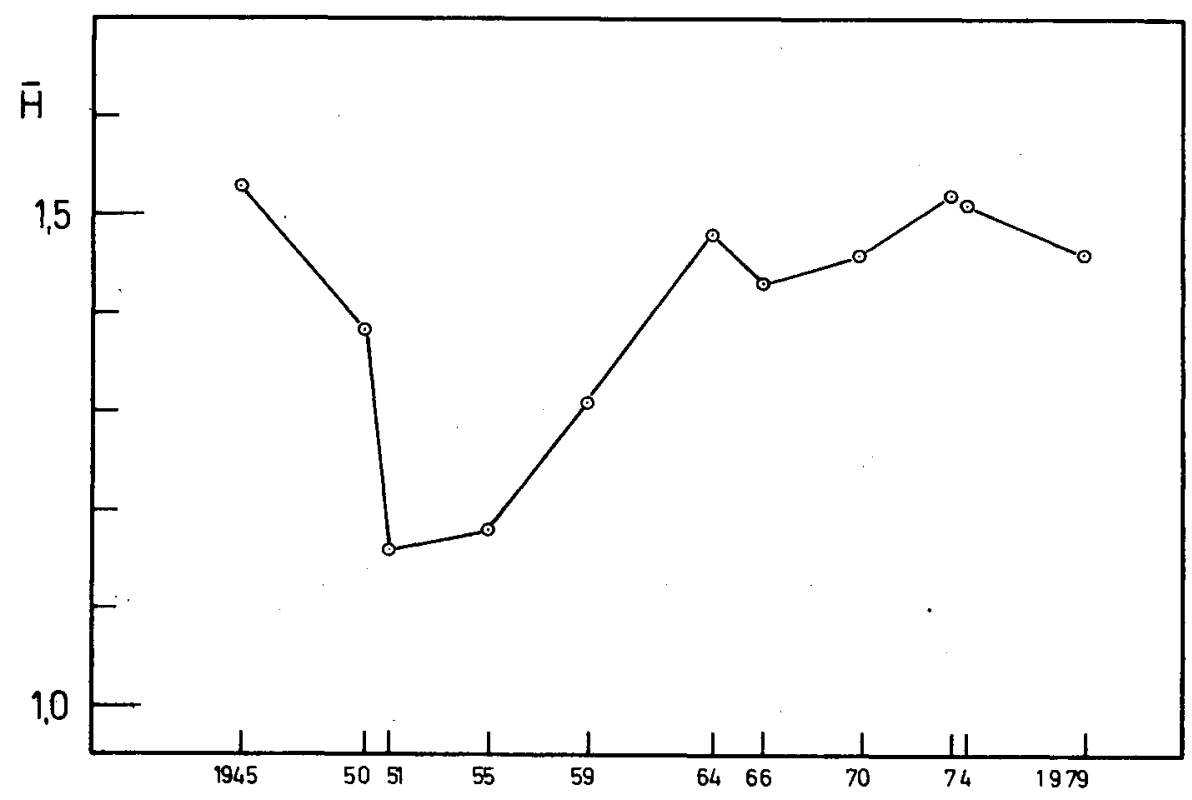

Gráfico 3.-Gran Bretaña. Elecciones a la Cámara Baja. Diversidad $(\bar{H})$ en bits/voto. 


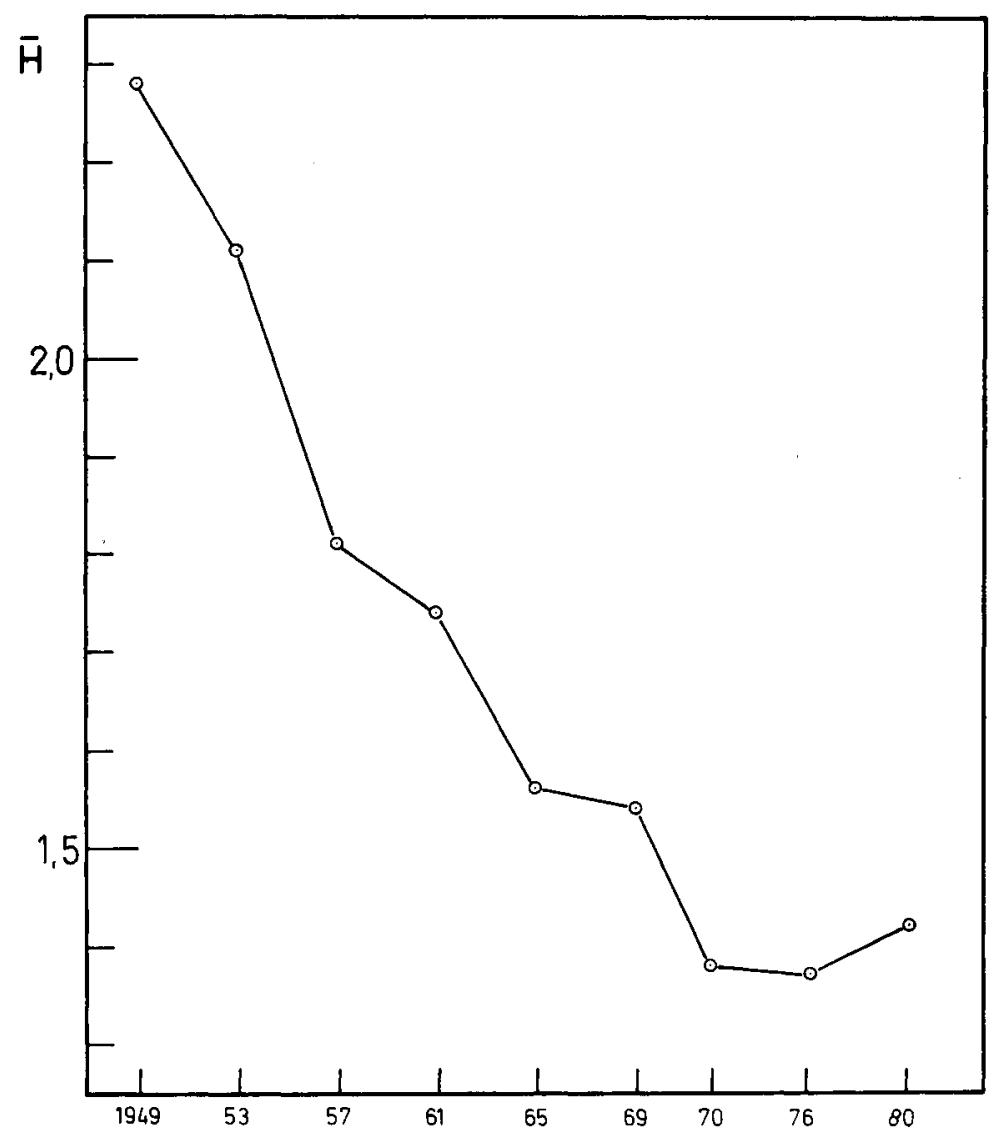

Gráficos 4--República lederal de Alemania. Ilecciones al Bundestag. Diversidad $(\bar{H})$ en bits/voto.

Se incluyen también gráficas de los casos de Israel y Suiza (gráficos 5 y 6) en el mismo período, pero que no participan de las mismas circunstancias históricas. Por ello no hay que esperar que los resultados se ajusten a modelo alguno de sucesión, como efectivamente no lo hacen. En el primer caso, Israel, procede señalar que las dos fuertes caídas de la Diversidad, años 69 y 81, suceden, respectivamente, tras la «Guerra de los Seis Días» (1967) y tras la firma del tratado de paz con Egipto y la retirada del Sinaí. Se diría que los períodos de crisis provocan una mesurable «bipolarización» del electorado. Por cierto que esta tendencia también podría constatarse en el caso inglés, en el que se advierten peculiares descensos de la Diversidad cuando hay «elecciones anticipadas» (períodos 50-51, 64-66, 74-74). 


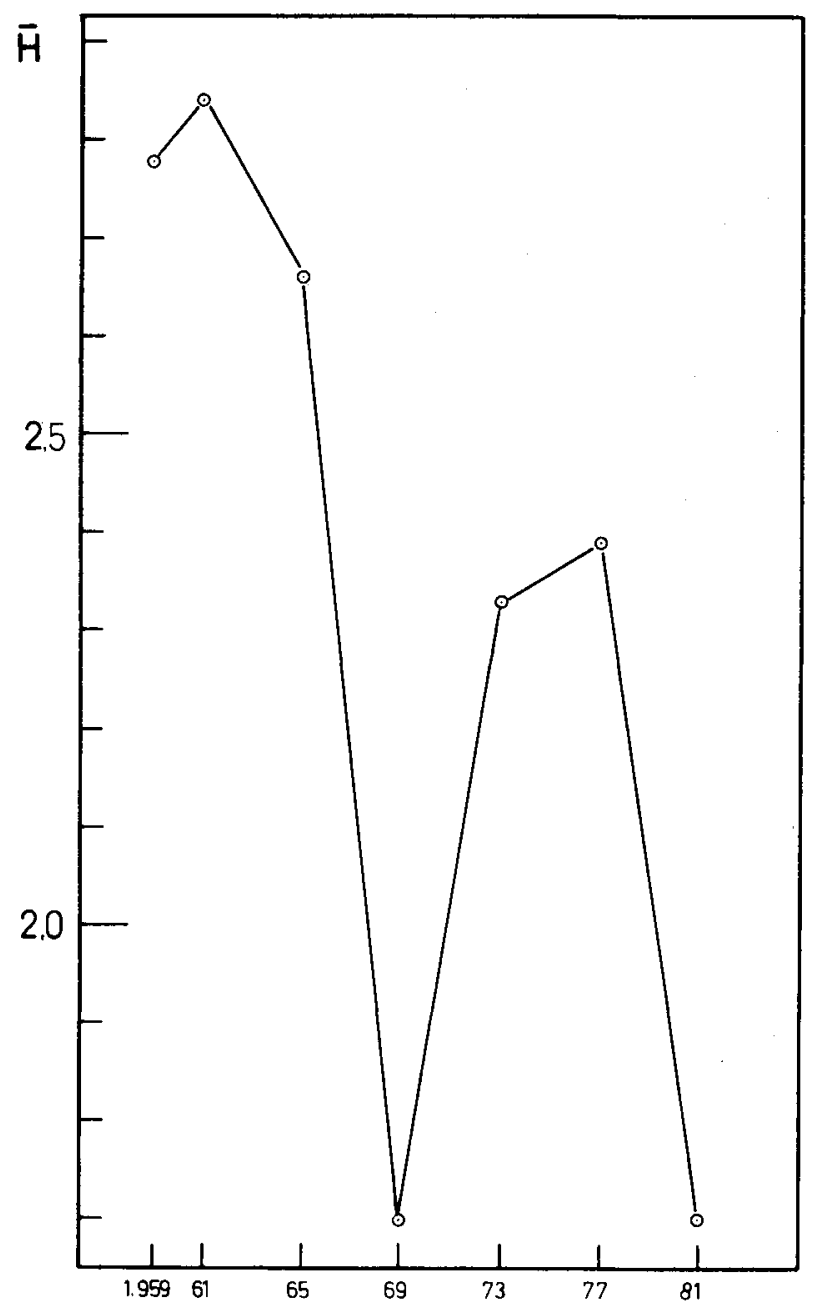

Gráfico 5.--Israel. Elecciones al Knesseth. Diversidad $(\bar{H})$ en bits/voto.

En cuanto a Suiza, se trata de un país que podríamos considerar en una «Fase Madura» asentada de años. Se advierte una fluctuación de amplitud más suave que en los casos anteriores y unos valores globales muy altos, lo que podría interpretarse como resultado de su peculiar cantonalismo (lo que los ecólogos llaman «efecto de Frontera») o bien de su antigüedad ininterrumpida.

Finalmente, el caso español, en el que se ha aplicado el método a tres 


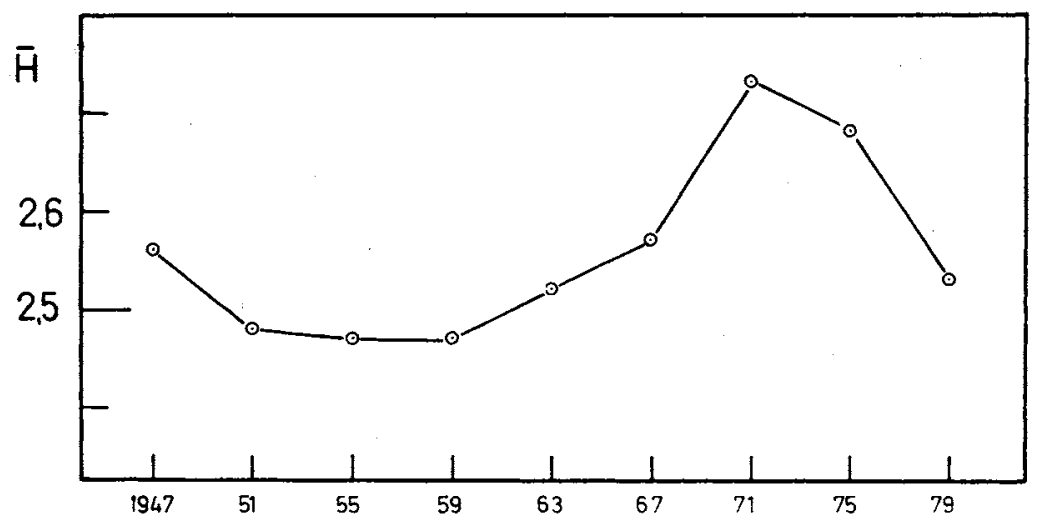

Gráfico 6.-Suiza. Elecciones al Consejo Nacional. Diversidad $(\bar{H})$ en bits/voto.

niveles: Barcelona, Cataluña y España (gráfico 7). Para el conjunto de todo el Estado, en las tres elecciones generales habidas hasta ahora, los resultados concuerdan con el modelo en su Fase II, es decir, la de "Ajuste», con un descenso continuado, como era de esperar en el corto periodo electoral habido. Destacan los bajos valores globales, así como lo amortiguado del descenso, tal vez como resultado de una situación previa latente muy prolongada, y por lo tanto «no tan latente».

El modelo parece cumplirse también para los casos de Barcelona y de Cataluña, en los que, impropiamente, se han incluido, además, las elecciones autonómicas de 1980. Impropiamente porque los votantes pueden no hacerlo de la misma forma si se trata de unas elecciones generales para todo el Estado que si son para su propia Comunidad. Esta discordancia puede explicar el que la diversidad global de toda Cataluña, en tales elecciones, sea mayor que la de Barcelona-ciudad, contra la norma en las otras votaciones. Por otro lado, el que tanto el valor de la Diversidad como su variación sean mayores en Barcelona que en Cataluña, y en éstas que en toda España, parece indicar una correlación del fenómeno descrito con la concentración demográfica y/o urbana. En este sentido se intentó buscarla, por municipios, entre la Diversidạd y el tamaño de la población, sin obtener resultados aparentes, al menos a primera vista. Intuitivamente resulta probable que tal correlación exista, pero no sólo con la población por sí sola, sino corregida con otros indicadores sociológicos, como podría ser el índice de inmigración u otros más elaborados. Sería necesario un trabajo más largo e intenso en tal dirección, que seguramente pondría de manifiesto tales correlaciones. Por ejemplo, muy probablemente, transcrita sobre un mapa, la Diversidad Política resultase un parámetro topografiable en virtud de un previsible efecto de contagio; la distribu- 


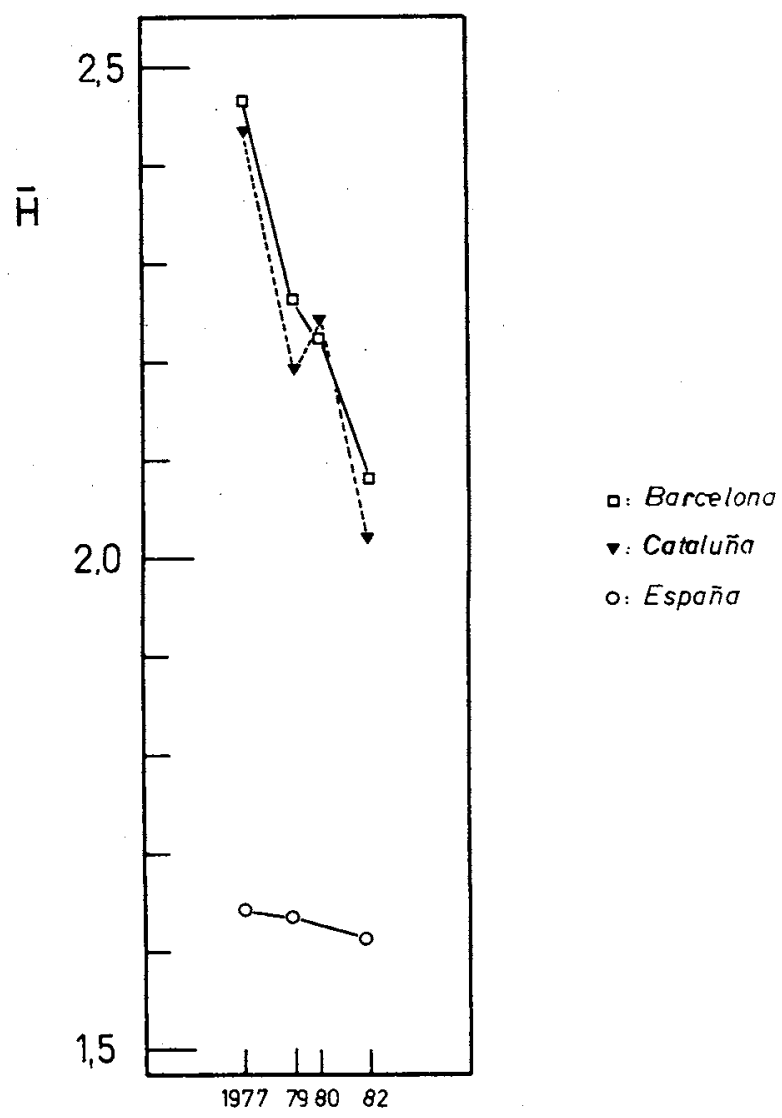

Gráfico 7.-España. Elecciones Generales y Autonómicas. Diversidad $(\bar{H})$ en bits/voto.

ción de zonas de alta y baja Diversidad, con seguridad habrá de estar en concordancia con la distribución de otros parámetros de interés ecosociológico. En la esperanza de haber aportado una idea fructífera a los sociólogos y/o a los ecólogos, no gueda sino expresar debido agradecimiento a los Departamentos de Ecología y de Derecho Político de la Universidad Autónoma de Barcelona, con cuya colaboración fue posible realizar este trabajo.

\section{Resumen}

Cuando los partidos políticos compiten por ocupar un espacio electoral recién constituido, se produce una situación análoga a la competencia entre 
las distintas especies vegetales por ocupar el territorio de un ecosistema. La dinámica de las proporciones relativas con las que tales especies se van distribuyendo es caracterizada por los ecólogos con el parámetro «diversidad», que, al estar calculado en base a tales proporciones relativas, puede también aplicarse a la diversidad de partidos en un espectro electoral. Haciéndolo en los compases iniciales de estructuración de un sistema político, se encuentra que su variación pasa por unas fases características análogas a las que se observan en la estructuración de un ecosistema, fenómeno que los ecólogos llaman precisamente «sucesión», y del que, en consecuencia, es posible hacer un modelo general para sistemas, basado simplemente en la limitación del espacio disponible, y en la competencia.

\section{BIBLIOGRAFIA EN CASTELLANO}

Margalef, Ramón: La Bios/era. Entre la Termodinámica y el juego, Omega.

- Ecologia, Omega.

Prigogine, Ilya: ¿Tan sólo una ilusión? Una exploración del caos al orden, Tusquets Editores.

Wagensberg, Jorge: Ideas sobre la complejidad del Mundo. Tusquets Editores. 
CRITICA DE LIBROS 